\title{
PATHOLOGY OF ANTERIOR POLIO-MYELITIS
}

\author{
By O. H. WILSON, M.D., \\ Nashville, Tenn.
}

From an historical point of view, the development of our present knowledge of the pathology of anterior polio-myelitis is quite interesting. Cornil, in $\mathrm{I}^{86} 6_{3}$, basing lis deductions on the postmortem examination of cases dying of subsequent disease, described this condition as one of primary cell degeneration, having its selective action upon the gray matter of the anterior loorns and nerve fibers arising there. In is7o, Rogers and Damaschino, after examining some acutely fatal specimens, demonstrated the inflammatory nature of the process, and consequent degeneration of nerve cells, still claiming its restriction to the anterior horns. While there has been much subsequent work upon the subject, this has been the accepted pathology; and is so stated in most books.

Recent writers have uniformly accepted the careful researches made by Harbitz and Scheel. of Christiana. during the Norwegian epidemic of 1905 and 1906 as the basis for the established pathology of polio-myelitis, which is especially gratifying, as it conforms to the new light upon the etiology and probable bacteriology. The exhaustive experiments upon monkeys, made by Flexner and Lewis, of the Rockefeller Institute, have done much to elucidate the subject, proving beyond doubt that the disease is due to an infection with some living reproducing organism, probably ultra microscopic in size, as is shown by the ability to transmit even through twenty-five generations, by intracranial injections of the filtrate of the emulsified diseased spinal cord, thus proving beyond doubt, by its intensified action, that it is bacterial, and not a toxin. This nullifies the patient toil of many preceding observers, who, recognizing from the epidemic character of the disease that it was probably of bacterial origin, had spent much time in searching the causative organism. Another cause for failure in investigations has been the negative results in working with the cerebrospinal fluid, which is perfectly clear and negative throughout the disease except, as Flexner and Lewis have shown in experiments upon monkeys, it becomes turbid two days before paralysis, that is, before we can recognize the condition.

Accepting the approved definition that the disease is a general infection, we might expect involvement of other viscera, even though it reserves its selective action for the cerebrospinal centers. The findings outside have been disappointing. Considering the probable modes of infection, we might look for postnasal and intestinal lesions, though it is probable that these have vanished before the case terminates fatally: Heart, ijver, kidneys, etc., have been accused of unusual conditions, but they are not constant. The Thymus gland is unusually active, but the period of greatest morbidity, the second year, represents a period of rapid change in this organ. That the discase is a general infection is shown by the toxicity of a lymphnode and the blood as demonstrated by Flexner and Lewis.

Harbitz and Scheel discuss the firdings in the central nervous system under two lreads, first in patients dying in the acute stage in the height of the diseased process; secondly, in those cases dying after defervescence of acute symptoms. Basing their deductions upon seventeen acute cases, they thus describe the condition. The microscopic findings are disappointing. The cord is congested, especially along the anterior portions and anterior horns with an occasional small hemorrhagic infarct; this condition is exaggerated at the lumbar and cervical swellings, though generally diminishing in intensity as you go up.

*Read before the Section on Medicine of the Southern Medical Association, Nashville, Tenn., November 8-10, 1910. 
Microscopically, we find a diffuse infiltrating inflammatory process, following the blood vessels, with proilferation of cells into the perivascular lymph spaces, most marked in the highly vascular regions, hence the more deciled involvement of the anterior horn. Nor is this process confined to the cord. It is coextensive with the pia mater and its prolongations, the microscopical condition being one of general hyperaemia, with occasional slight hemorrhages, and cell infiltration, accentuated in the anterior horn. It is probable that the pia is first involved, as the cord under it may not yet show signs of inflammation. With this meningitis probably comes the infection of the cerebro-spinal fluid, and then extension along the vessels to the cord. No part of the pia is entirely free from inflammatory products. That covering the medulla and pons is always involved. Then the meninges of the base, then along the floor of the fourth ventricle to the sylvian fossa, involving neuclei of all the cranial nerves. and even the deeper ganglia, reaching them by the blood vessels. Even the pia on the cerebral cortex is affected. Harbitz and Scheel make the statement that in scuerc or fatal cases, we have, as a rule, diffuse inflammation of the entire cord with its pia, of the entire medulla and pons, of the basal ganglia, and often also of the cortex of the brain to a greater or less extent, always in connection with a similar inflammation of the pia. Nearly all fatal cases terminate by paralysis of respiration, and in these we find oedema of the floor of the fourth ventricle, with an extension of the inflammatory process well up the sylvian acqueduct. The destruction of the ganglionic cell is a secondary resuit of the inflammatory process and the cell infiltration. The activity of the inflammation and the resistance of tissue determines the extent of this, which of course determines the character of the permanent paralysis.

Such is the picture of an anterior polio-myelitis infection, the real disease, tissue resistance and intensity of the inflammation determining the location of the resulting paralysis.
The actual infection and the inflammatory condition is generally coextensive with the pia, the weaker ganglionic cells with the greatest inflammatory exudate marking the extent and location of the paralysis, hence the symptoms. and nomenclature, all parts of the same process. Strumpell many years ago suggested the probable etiologic connection between diseased processes in widely different localities. in the nervous system.

The above findings were all in severe and rapidly fatal cases, dying when lesions were at their height. Harbitz and Scheel also examined four cases dying of intercurrent conditions, when the cerebro-spinal process was in. the stage of repair, and find, even here, evidences of the same inflammatory process all through various parts of the pia, showing that even in milder cases there is the same extensive inflammation.

The resulting changes in the cord during the process of repair have long been known. The ventral horn is much atrophied, ganglionic cells are degenerated and diminished in number. Certain fibers of the anterior nerves. have disappeared, there still exist infiltrated round cells, evidences of the inflammation. The muscles supplied by affected nerves becomeflaccid, atrophied, possibly resulting in unapposed contractures. In children with extensive paralysis there is trophic change in thebone, resulting in stunting the limb.

The burden of this paper is to show that: anterior polio-myelitis is an infectious disease, affecting the pia mater of practically the entire. central nervous system. That the actual infection is accompanied by cell proliferation. into the perivascular lymph spaces, the amount and Jocation of said cell proliferation due tothis infection, together with tissue resistance, that is, the ability of the nerve tissue to take care of the products of inflammation, determine the type of the disease. When all is. absorbed with no appreciable ganglionic destruction, we have the so-called abortive type. When we find localized destriction of some cells in the anterior horn, chiefly in the lum- 
bar enlargement, we have the common type of polio myelitis. When the pathology is localized and intense at a point involving practically the entire section of the cord, a rare condition, we may have transverse myelitis. Acute bulbar paralysis may be seen winen the intensity of the disease is located in that region. Encephalitis when the cord symptoms are insignificant. Polio-encephalitis when the symptoms are general. Why could we not explain, as Harbitz and Scheel seem to think we should, Landrys ascending paralysis as part of the same process.

\title{
SYMPTOMS AND DIAGNOSIS OF ANTERIOR POLIO-MYELITIS
}

\author{
By CHARLES M. NICE, M.D., \\ Pediatrician to St. Vincent's Hospital, \\ Birmingham, Alabama.
}

The progress and advancement of medical knowledge is liberally shown in the symptomatic and diagnostic study of this disease, anterior polio-myelitis. It is true that the laboratory has thus far outclassed the clinical side, yet a full consideration of the reports which have come from recent epidemics is extremely interesting, especially when studied with th:e classical text-book as a corollary.

Numerous names have been suggested to better meet the demands of our present knowledge, just as with classifications which have been sought to correspond with the varied determination of symptoms. For the purpose of study, the writer would like to follow 'tlu: general custom and suggest that the nethe. cercbro-spinal polio-myclitis be substituted for the nany now in use, believing that it more nearly meets the pathological and clinical aspects as they are presented to us.

As you know, the discase is most common in infants and childhood, though the elderly are not excepted. It occurs in the summer and fall months, the winter in some way appearing to be a factor in dissipating the infection.

As a working classification, the disease may well be considered as appearing under three types: (a) mild, (b) severe, and (c) abortive. Any or all of these may occur in the same epidemic or even in the same household. In the mild type, the symptoms may be so mild as to escape unnoticed by the family or attendants and the true nature of the affection only. discovered after disability or paralysis has occurred. Even the paralysis may affect only a muscle group or two, and the recovery be so rapid that the afflicted one is able to walk or be around within a few days. The scucrelype may end its subject by death within a short time after the onset. It is associated with suddenness, great pain, fever, prostration and perhaps with uncontrollable convulsions, dyspnoea and coma. The resulting paralysis may be of bulbar origin, giving pneumogastric distribution, always to be considered with gravity. Or there may be extensive and irreparable paralysis leading to marked atrophies and deformities. The abortive type may display either of the above characteristics, but with an abrupt ending of recovery and without paralysis.

The symptoms may be further divided into cercbral, spinal and ncuritic, or there may be any combination of these irrespective of the type of disease. These terms are used to designate the characteristics of the preponderant symptoms in a given case. In the first instance, the onset is sudden, with severe headache, prostration, possibly delirium, convulsions, a-retracted head and the presence of a Kernig's sign. In the second instance, the onset may be just as sudden, but the symptoms

*Read before the Section on Medicine of the Southem Medical Association at Nashville, Ten., November 8, 9, 10, 1910. 